\title{
Conflict-Driven Conditional Termination
}

\author{
Vijay D'Silva ${ }^{1}$ and Caterina $\operatorname{Urban}^{2(\otimes)}$ \\ 1 Google Inc., San Francisco, USA \\ 2 École Normale Supérieure, Paris, France \\ urban@di.ens.fr
}

\begin{abstract}
Conflict-driven learning, which is essential to the performance of SAT and SMT solvers, consists of a procedure that searches for a model of a formula, and refutation procedure for proving that no model exists. This paper shows that conflict-driven learning can improve the precision of a termination analysis based on abstract interpretation. We encode non-termination as satisfiability in a monadic second-order logic and use abstract interpreters to reason about the satisfiability of this formula. Our search procedure combines decisions with reachability analysis to find potentially non-terminating executions and our refutation procedure uses a conditional termination analysis. Our implementation extends the set of conditional termination arguments discovered by an existing termination analyzer.
\end{abstract}

\section{Conflict-Driven Learning for Termination}

Conflict-driven learning procedures are integral to the performance of SAT and SMT solvers. Such procedures combine search and refutation to determine if a formula is satisfiable. Conflicts discovered by search drive refutation, and search learns from refutation to avoid regions of the search space without solutions.

Our work is driven by the observation that discovering a small number of disjunctive termination arguments is crucial to the performance of certain termination analyzers [27]. Figure 1 summarizes our lifting of conflict-driven learning to termination analysis. We use reachability analysis to find a set of states that constitute potentially non-terminating execution. We apply a conditional termination analysis to this set to eliminate states from which all executions terminate. Unlike termination analysis, which solves a decision problem and returns a YES or NO answer, conditional termination analysis is concerned with discovering sufficient conditions for termination. Sufficient conditions for termination play the role of learned clauses in our analysis. They prevent subsequent runs of reachability analysis from revisiting states from which termination is guaranteed.

Our conflict driven conditional termination procedure ( $\mathrm{CDCT}$ ) can be viewed as a sound but incomplete solver for a family of monadic, second-order formulae. Büchi's theorem shows that the language of a Büchi automaton is non-empty exactly if a formula in the monadic second-order theory of one successor (s1s) is satisfiable [5]. This theorem can be viewed encoding non-termination of a finitestate program as satisfiability in s1s. We introduce $\mathrm{S} 1 \mathrm{~S}(\mathrm{~T})$, an extension of $\mathrm{S} 1 \mathrm{~S}$

(C) Springer International Publishing Switzerland 2015

D. Kroening and C.S. Păsăreanu (Eds.): CAV 2015, Part II, LNCS 9207, pp. 271-286, 2015.

DOI: $10.1007 / 978-3-319-21668-3 \_16$ 


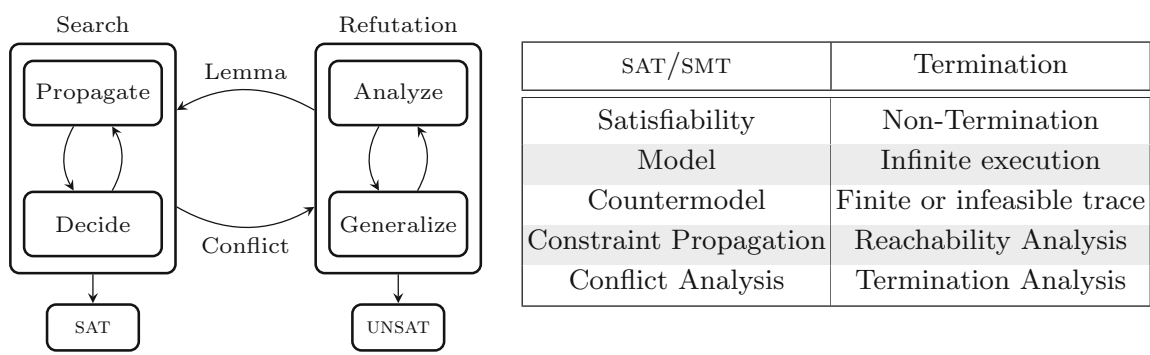

Fig. 1. Conflict driven learning as applied to termination

to sequences of first-order structures, and encode non-termination in a controlflow graph ( $\mathrm{CFG}$ ) as satisfiability in $\mathrm{s} 1 \mathrm{~s}(\mathrm{~T})$. A model of a formula is an infinite execution that respects the transition constraints in the CFG.

Formulating non-termination as satisfiability provides a clear route for lifting CDCL to non-termination. We combine decisions with reachability in an abstract domain to construct and refine assignments to second-order variables in the same way that SAT solvers construct and refine partial assignments. A notable difference to standard abstract interpretation is that our assignments are neither over- nor under-approximations of the set of reachable states. Our conflict analysis uses backwards abstract interpretation to enlarge the set of states from which termination is guaranteed. We present a generalized unit rule for combining ranking functions with reachability analysis. These components are combined in our new analysis, which we have implemented and evaluated against state-of-the-art termination provers.

\section{Non-Termination as Second-Order Satisfiability}

The two contributions of this section are the logic s1s(T), which extends the monadic second-order logic of one successor (S1s) with a theory and an encoding of program non-termination as satisfiability in this theory.

\subsection{Monadic Second-Order Theories of One Successor}

We use $\hat{=}$ for definition. Let $\mathcal{P}(S)$ be the powerset of $S$. For $f: A \rightarrow B$, the function $f[a \mapsto b]$ maps $a$ to $b$ and $c$ distinct from $a$ to $f(c)$. The symbols $x, y, z$ range over first-order variables in Vars, $f, g, h$ over functions in Fun, and $P, Q, R$ over predicates in Pred. We use a set Pos of first-order position variables whose elements are $i, j, k$, a set $S V a r$ of monadic second-order variables denoted $X, Y, Z$, a unary successor function suc and a binary successor predicate $S u c$.

Our logic consists of three families of formulae called state, transition and trace formulae, which are interpreted over first-order structures, pairs of firstorder structures and infinte sequences of first-order structures respectively. The formulae are named after how they are interpreted over programs. 


$$
\begin{aligned}
& t::=x \mid f\left(t_{0}, \ldots, t_{n}\right) \\
& \varphi::=P\left(t_{0}, \ldots, t_{n}\right)|\varphi \wedge \varphi| \neg \varphi \\
& \psi::=\operatorname{suc}(x)=t|\psi \wedge \psi| \neg \psi \\
& \Phi::=X(i)|\operatorname{Suc}(i, j)| \varphi(i) \mid \psi(i) \\
& |\operatorname{|r}| \neg \Phi \mid \exists i: \operatorname{Pos} . \Phi
\end{aligned}
$$

Term

State Formula

Transition Formula

Trace formula

A first-order interpretation $(\mathrm{Val}, I)$ defines functions $I(f)$ and relations $I(P)$ over values in Val. The value $\llbracket t \rrbracket_{s}$ of a term $t$ in a state $s: \operatorname{Vars} \rightarrow \operatorname{Val}$, is $s(x)$ if $t$ is $x$, and $I(f)\left(\llbracket t_{0} \rrbracket_{s}, \ldots, \llbracket t_{n} \rrbracket_{s}\right)$ if $t$ is $f\left(t_{0}, \ldots, t_{n}\right)$. The interpretation of a state formula is the standard first-order semantics. A transition formula is interpreted at a transition, that is, a pair of states $(r, s)$. A formula $\varphi$ in which the symbol suc does not occur is interpreted at the state $r$, while $s u c(x)=t$ compares the value of the term $t$ in $r$ with the value of $x$ in the successor state $s$.

$$
\begin{aligned}
& (r, s) \models P\left(t_{0}, \ldots, t_{n}\right) \text { if }\left(\llbracket t_{0} \rrbracket_{r}, \ldots, \llbracket t_{n} \rrbracket_{r}\right) \in I(P) \\
& (r, s) \models \varphi \wedge \psi \text { if }(r, s) \models \varphi \text { and }(r, s) \models \psi \\
& (r, s) \models \neg \varphi \text { if }(r, s) \vDash \varphi \quad(r, s) \models s u c(x)=t \text { if } \llbracket x \rrbracket_{s}=\llbracket t \rrbracket_{r}
\end{aligned}
$$

A trace $\tau: \mathbb{N} \rightarrow$ (Vars $\rightarrow$ Val) is an infinite sequence of states and $\tau(m)$ is the state at position $m$. A position assignment $\sigma:($ Pos $\rightarrow \mathbb{N}) \cup(S \operatorname{Var} \rightarrow \mathcal{P}(\mathbb{N}))$ maps position variables to $\mathbb{N}$ and second-order variables to subsets of $\mathbb{N}$ such that $\{\sigma(X) \mid X \in S \operatorname{Var}\}$ partitions $\mathbb{N}$. We explain this partition condition shortly. A trace formula is interpreted with respect to an $\operatorname{sis}(\mathrm{T})$ structure $(\tau, \sigma)$.

Note that there are first-order variables of two sorts in a trace formula. A trace formula $\Phi$ asserting that the transition formula $\psi(x, y) \hat{=} \operatorname{suc}(x)=y+1$ is true at the trace position denoted by $i$ has the form $\psi(x, y)(i)$. The predicate $\operatorname{Suc}(i, j)$ asserts that the position $j$ occurs immediately after $i$.

$$
\begin{array}{ll}
(\tau, \sigma) \models S u c(i, j) \text { if } \sigma(i)+1=\sigma(j) & (\tau, \sigma) \models \varphi(i) \text { if } \tau(\sigma(i)) \models \varphi \\
(\tau, \sigma) \models \psi(i) \text { if }(\tau(\sigma(i)), \tau(\sigma(i)+1)) \models \psi & (\tau, \sigma) \models X(i) \text { if } \sigma(i) \in \sigma(X) \\
(\tau, \sigma) \models \Phi \wedge \Psi \text { if }(\tau, \sigma) \models \Phi \text { and }(\tau, \sigma) \models \Psi & (\tau, \sigma) \models \neg \Phi \text { if }(\tau, \sigma) \models \Phi \\
(\tau, \sigma) \models \exists i: \operatorname{Pos} . \Phi \text { if }(\tau, \sigma[i \mapsto n]) \models \Phi & \text { for some } n \text { in } \mathbb{N}
\end{array}
$$

An $\operatorname{Sis}(\mathrm{T})$ structure $(\tau, \sigma)$ is a model of $\Phi$ if $(\tau, \sigma) \models \Phi$, and is a countermodel otherwise. A trace formula is satisfiable if it has a model. An $\operatorname{sis}(\mathrm{T})$ structure is defined using an infinite trace, so finite traces cannot be models of a formula.

\subsection{Encoding Non-Termination in S1S(T)}

We now recall control flow graphs (CFGs) and encode non-termination as satisfiability. A command in $C m d$ is an assignment $x:=t$ of a term $t$ to a firstorder variable $x$, or is a condition $[\varphi]$, where $\varphi$ is a state formula. A CFG $G=(L o c, E$, in, ex, stmt) consists of a finite set of locations Loc including an 


$$
\begin{gathered}
\left(\forall i . F i r s t(i) \Rightarrow X_{\text {in }}(i)\right) \wedge\left(\forall i . X_{\mathrm{ex}}(i) \Rightarrow \operatorname{Last}(i)\right) \\
\wedge \forall i . \forall j . X_{\text {in }}(j) \wedge \operatorname{Suc}(i, j) \Rightarrow(\operatorname{suc}(x)=x-1)(i) \wedge X_{\mathrm{a}}(i) \\
\wedge \forall i . \forall j . X_{\mathrm{a}}(j) \wedge \operatorname{Suc}(i, j) \Rightarrow(x \neq 0 \Rightarrow \operatorname{suc}(x)=x)(i) \wedge X_{\text {in }}(i) \\
\wedge \forall i . \forall j . X_{\mathrm{ex}}(j) \wedge \operatorname{Suc}(i, j) \Rightarrow(x=0 \Rightarrow \operatorname{suc}(x)=x)(i) \wedge X_{\text {in }}(i)
\end{gathered}
$$

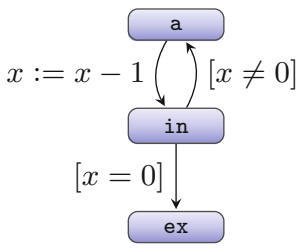

Fig. 2. A formula encoding non-termination of the program shown in the monadic second-order theory of one successor over integer arithmetic.

initial location in, an exit location ex, edges $E \subseteq L o c \times L o c$, and a labelling stmt $: E \rightarrow C m d$ of edges with commands. To assist the presentation, we assume that the exit location ex has no successors.

The formula Trans $_{c}$ below defines the semantics of commands using the condition Same $_{V} \hat{=} \bigwedge_{x \in V} \operatorname{suc}(x)=x$, that variables in $V$ are not modified. The set of models of Trans $_{c}$ is the transition relation Rel $_{c}$. We write Trans $_{e}$ and $\mathrm{Rel}_{e}$ for the transition formula and relation of the command stmt $(e)$. The formula $\operatorname{Inf}_{G}$ extends the translation of Büchi automata to s1s to encode CFGs in $\operatorname{sis}(\mathrm{T})$. We write $\operatorname{First}(i) \hat{=} \forall j . \neg S u c(j, i)$ for the first position on a trace and $\operatorname{Last}(i) \hat{=} \forall j . \neg \operatorname{Suc}(i, j)$ for a position that cannot be on an infinite trace.

$$
\begin{aligned}
\text { Trans }_{c}= & \begin{cases}b \Longrightarrow \text { Same }_{\text {Vars }} & \text { if } c=[b] \\
\operatorname{suc}(x)=t \wedge \text { Same }_{\text {Vars } \backslash\{x\}} & \text { if } c=x:=t\end{cases} \\
\operatorname{Inf}_{G} \hat{=} & \left(\forall i . \text { First }(i) \Longrightarrow X_{\text {in }}(i)\right) \wedge\left(\forall j . X_{\mathrm{ex}}(j) \Longrightarrow \operatorname{Last}(j)\right) \\
& \wedge \bigwedge_{\mathrm{v} \in \operatorname{Loc}} \forall i . \forall j . X_{\mathrm{v}}(j) \wedge \operatorname{Suc}(i, j) \Longrightarrow \bigvee_{(\mathrm{u}, \mathrm{v}) \in E} \operatorname{Trans}_{(\mathrm{u}, \mathrm{v})}(i) \wedge X_{\mathrm{u}}(i)
\end{aligned}
$$

The formula $I n f_{G}$ encodes program behaviour as follows. Consider an $\mathrm{s} 1 \mathrm{~s}(\mathrm{~T})$ structure $(\tau, \sigma)$. The interpretation $\sigma\left(X_{\ell}\right)$ of a second-order variable $X_{\ell}$ represents positions on the trace when execution is at location $\ell$. Such an interpretation partitions $\mathbb{N}$ because each position on a trace corresponds to a unique location. The entry constraint on First $(i)$ ensures execution begins at in. The exit constraint implying Last $(j)$ enforces that an infinite execution does not visit ex. The conditions involving $\operatorname{Suc}(i, j)$ are called transition constraints and express that consecutive states on a trace must respect the transition relation of $G$. Theorem 1 expresses non-termination as satisfiability.

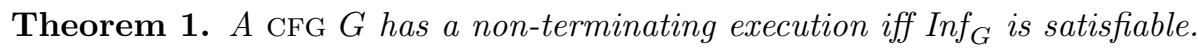

We believe this is a simple yet novel encoding of non-termination that allows the duality between search and refutation to be exploited for termination analysis. In contrast, the second-order encoding of termination in [13] uses a predicate for disjunctive well-foundedness and is solved in a different manner.

Example 1. A CFG $G$ and the formula $I n f_{G}$ for a program with a variable $x$ of type $\mathbb{Z}$ are shown in Fig. 2. We write a trace as a sequence of values of $x$. Let $\tau$ 
be the trace $-1,-1,-2,-2, \ldots$ and $\sigma$ the assignment mapping $X_{\text {ex }}$ to the empty set, and $X_{\text {in }}$ and $X_{\mathrm{a}}$ to even and odd positions, respectively. The structure $(\tau, \sigma)$ is a model of $I n f_{G}$. Every structure $(\tau, \delta)$, with $\tau$ as before, in which $\delta\left(X_{\text {ex }}\right)$ is not empty is a countermodel of $I n f_{G}$ because ex is not reachable if $x$ is initially -1 , so some transition in $\tau$ must violate a transition constraint in $\operatorname{Inf}_{G}$. Every structure $\left(\tau^{\prime}, \delta^{\prime}\right)$ with $x$ non-negative in $\tau^{\prime}(0)$ is also a countermodel of Inf $_{G}$ because executions with $x$ initially non-negative terminate. Since $\tau^{\prime}$ is infinite by definition, some transition in $\tau^{\prime}$ must be infeasible. Terminating executions cannot be models of $\operatorname{Inf}_{G}$ because traces in $\mathrm{s} 1 \mathrm{~s}(\mathrm{~T})$ structures are infinite. $\triangleleft$

The formula $\operatorname{Inf}_{G}$ is a conjunction of formulae in which second-order variables and first-order program variables are free but first-order position variables are bound. We exploit this structure in our analysis.

\section{Conflict-Driven Conditional Termination}

The conflict-driven conditional termination procedure (CDCT) in Algorithm 1 generalizes CDCL from SAT to termination analysis. The input is the formula $\operatorname{Inf} f_{G}$. The output (result, $\Delta, \Theta$ ) is a result concerning a set of structures $\Delta$ and a set $\Theta$ of piecewise-defined ranking functions (PDRFs).

The value of result is one of divergent, terminates, or unknown. CDCT returns divergent if the traces represented by $\Delta$ do not reach the exit location, which could be due to non-termination or undefined behaviour; It returns terminates if $\Delta$ is empty and $\Theta$ guarantees termination for all states. It returns unknown if CDCT cannot prove termination and cannot progress. This happens if the abstract domain cannot accurately represent non-terminating executions, if the ranking functions used cannot express a termination argument, or a bound on the number of decisions has been exceeded.

CDCT maintains four global data structures. The trail $t r$ is a sequence of assignments to second-order variables. The explanation array exp contains in each element $\exp [i]$, the decision or constraint used by propagation to add $\operatorname{tr}[i]$ to the trail. The set of PDRFs $\Theta$, generated by conditional termination analysis, are our analogue of learned clauses. The blocking constraints $\Psi$ contain constraints representing two types of states, which need not be revisited. One is states from which all executions terminate. The other is states for which CDCT could neither prove termination nor demonstrate non-termination.

Each execution of the CDCT loop begins with a call to Search(), which attempts to find a non-terminating execution. If Search() returns divergent, CDCT returns. If Search () returns unknown, the trail represents a potential conflict because it has discovered a set of states from which some execution terminates. The conflict is potential because the trail may also contain models of $\operatorname{Inf} f_{G}$. This is a difference to SAT and SMT solvers where a conflict contradicts a formula.

The conflict analysis procedure Analyze() extracts from a potential conflict a definite conflict $\theta$, expressed as a ranking function. The domain of $\theta$ represents 

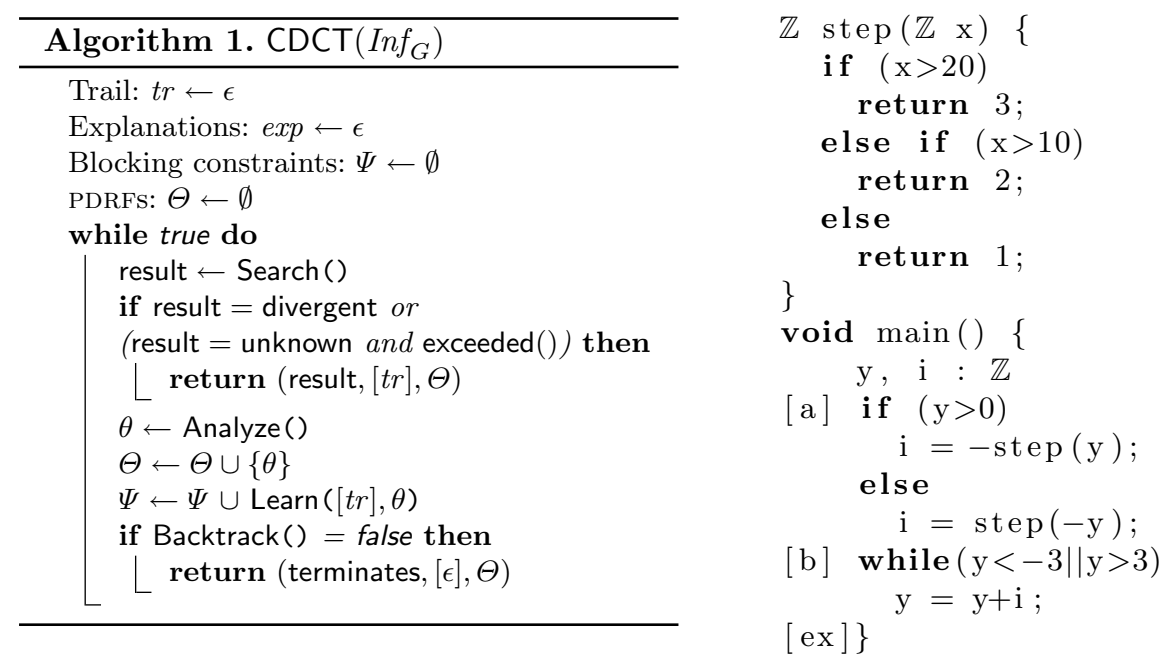

states from which all executions terminate. The learning step Learn() generates a blocking constraint to drive subsequent search away from these states. Learning also generates a blocking constraint if $\mathrm{CDCT}$ cannot make progress analyzing $[t r]$. This happens if no more decisions can be made and no ranking function can be extracted. CDCT then backtracks if possible.

An Example Run. A program is shown in C-like syntax alongside Algorithm 1. The location $\mathrm{a}$ is reached after the variables are initialized, $\mathrm{b}$ is the loop head, and ex is the exit location. The program terminates but the abstract interpretationbased tool FunCTION [32] cannot prove termination. CDCT enables FunCTION to prove termination while also avoiding case explosion. Even though other tools may be able to prove termination, we believe CDCT is interesting because similar ideas could be used to expand the programs handled by those tools.

In this example, we use an interval abstract domain and affine ranking functions. Search () uses reachability analysis to derive the intervals $y:[-3,3], i:[-3,3]$ at ex but termination analysis fails. Decisions restrict the range of a variable at a location: for example, Search() heuristically uses conditions from the code to make the decisions $y:[1, \infty]$ and $y:[-\infty, 10]$ at location a. Reachability derives the range $y:[1,3], i:[-1,-1]$ at ex, which is a conflict, because no trace with these states at ex satisfies $I n f_{G}$. Analyze() represents this conflict as $X_{\mathrm{ex}} \mapsto\{y:[1,3], i:[-1,-1] \rightarrow 0\}$, which assigns a PDRF to the second-order variable $X_{\text {ex }}$ and expresses that the program terminates in 0 steps for the states shown. The PDRF is propagated backwards through the program by an abstract interpreter [31] to derive the second-order assignments below. We omit the interval on $i$, which is unchanged.

$$
X_{\mathrm{ex}} \mapsto y:[1,3] \rightarrow 0, X_{\mathrm{b}} \mapsto y:[1,3] \rightarrow 1, X_{\mathrm{b}} \mapsto y:[4,4] \rightarrow 3, X_{\mathrm{b}} \mapsto y:[5,5] \rightarrow 5
$$


If these assignments are propagated to location $\mathrm{b}$, we could only prove that the program terminates for $y:[1,5]$ at a. Instead, we apply widening to the PDRFs to derive $X_{b} \mapsto\{y:[1,3] \rightarrow 1, y:[4,10] \rightarrow 2 x+5\}$, which bounds the number of steps to termination at the loop head for $y$ in the ranges shown. We heuristically expand the piece $y:[4,10]$ of the PDRF to $y:[1, \infty]$ and check if the $2 x+5$ is still a ranking function. Since it is, we have proved termination for executions with $y:[1, \infty], i:[-1,-1]$ at $\mathrm{b}$, despite having explicitly only analyzed the range $y:[0,5]$.

The learning step complements the decision $y:[1, \infty]$ and uses $X_{\mathrm{a}} \mapsto y:[-\infty, 0]$ to restrict future search. Learnt constraints typically have more structure. A similar run of CDCT can show termination when $y$ is initially non-positive.

Consider the program with the loop condition changed to $(y>-3)$. Now, the program does not always terminate. Decisions and learning can infer a ranking function for positive $y$ as before. Decisions can also discover that for $X_{\mathrm{a}} \mapsto$ $y:[-1,-1]$, ex is unreachable, indicating non-termination (as all locations lead to ex). In this way, CDCT proves conditional termination using disjunctions of ranking functions and also identifies non-terminating executions.

\section{Search for a Conflict}

We now show how a trail, a data structure used by SAT solvers, can be used to make explicit the incremental progress made by an abstract interpreter.

Abstract Domains. A bounded lattice $(L, \sqsubseteq, \sqcap, \sqcup)$ is a partially ordered set with a meet $\sqcap$, a join $\sqcup$, a greatest element $\top$ (top), and a least element $\perp$ (bottom). A concrete domain for forward analysis $(\mathcal{P}($ State $), \subseteq, F)$ is a lattice of states with a set $F=\left\{\right.$ post $\left._{c} \mid c \in C m d\right\}$ of monotone functions called transformers, where $\operatorname{post}_{c}(S)$ is the image of $S$ under the transition relation for $c$. An abstract domain is a bounded lattice $(A, \sqsubseteq, G, \nabla)$ with a set of abstract transformers $G=\left\{\right.$ post $\left._{c}^{A} \mid c \in C m d\right\}$ and a widening operator $\nabla: A \times A \rightarrow A$. There is a monotone concretization function $\gamma: A \rightarrow \mathcal{P}($ State $)$ satisfying that $\gamma(\top)=$ State and $\gamma(\perp)=\emptyset$. The transformers satisfy the soundness condition $\operatorname{post}_{c}(\gamma(a)) \subseteq$ $\gamma\left(\right.$ post $\left._{c}^{A}(a)\right)$ that abstract transformers overapproximate concrete transformers.

Literals are essential for propagation and conflict analysis in SAT. The analogue of literals in abstract domains are complementable meet-irreducibles [11]. A lattice element $c$ is a meet-irreducible if $a \sqcap b=c$ implies that $a=c$ or $b=c$. Let $\mathcal{M}_{A}$ be the meet-irreducibles of $A$. An abstract element a has a concrete complement if there exists an $\bar{a}$ in $A$ such that $\gamma(a)=\neg \gamma(\bar{a})$. A meet decomposition of an element $a$ is a finite set $m d c(a) \subseteq \mathcal{M}_{A}$ satisfying that $\Pi m d c(a)=a$ and that there is no strict subset $S \subset m d c(a)$ with $\prod S=a$. A has complementable meet irreducibles if every $m \in \mathcal{M}_{A}$ has a concrete complement $\bar{m} \in \mathcal{M}_{A}$.

Example 2. The interval lattice has elements $[a, b]$, where $a \leq b \in \mathbb{Z} \cup\{-\infty, \infty\}$. The intervals $[-\infty, k],[k, \infty]$ are meet-irreducibles, unlike $[0,2]$. The set $S=$ $\{[-\infty, 2],[0, \infty],[-5, \infty]\}$ satisfies $\prod S=[0,2]$ but is not a meet decomposition because $\{[-\infty, 2],[0, \infty]\} \subset S$. The concrete complements of $[-\infty, k]$ and $[k, \infty]$ are $[k+1, \infty]$ and $[-\infty, k-1]$, while $[0,2]$ has no concrete complement. 


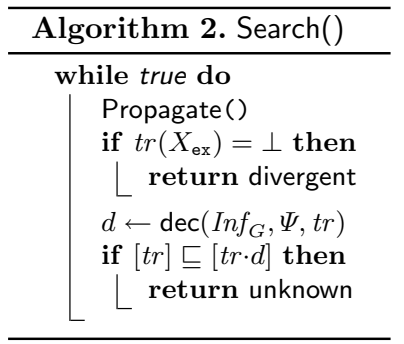

\begin{tabular}{|c|l|c|c|}
\hline & Trail $t r$ & $\exp$ & Modification \\
\hline \hline 1 & $\epsilon$ & & Initial state \\
2 & $X_{\text {ex }}:[-\infty, 0], X_{\text {ex }}:[0, \infty]$ & $\{$ in, a, ex $\}$ & Propagation \\
\hline \hline $3 \mathrm{a}$ & $\hookrightarrow X_{\text {in }}:[9, \infty] X_{\text {in }}:[0, \infty]$ & dec & Decision \\
$4 \mathrm{a}$ & $X_{a}:[1, \infty]$ & $\{\mathrm{a}$, in $\}$ & Propagation \\
$5 \mathrm{a}$ & $X_{\text {in }}:[-\infty, 0]$ & dec & Decision \\
$6 \mathrm{a}$ & $X_{\mathrm{a}}: \perp$ & $\{\mathrm{a}$, in $\}$ & Propagation \\
\hline \hline $3 \mathrm{~b}$ & $X_{\text {in }}:[-\infty,-7]$ & dec & Decision \\
$4 \mathrm{~b}$ & $X_{a}:[-\infty,-7], X_{\text {ex }}: \perp$ & $\{\mathrm{a}$, in $\}$ & Propagation \\
\hline
\end{tabular}

Abstract Assignments. SAT solvers use partial assignments to incrementally construct a model. We introduce abstract assignments, which use abstract domains to represent $\mathrm{s} 1 \mathrm{~S}(\mathrm{~T})$ structures. Let Struct be the set of $\mathrm{s} 1 \mathrm{~S}(\mathrm{~T})$ structures. The lattice of abstract assignments $\left(A s g_{A}, \sqsubseteq\right)$ contains the set $A s g_{A} \hat{=} S \operatorname{Var} \rightarrow A$ with the pointwise order: $a s g \sqsubseteq a s g^{\prime}$ if $a s g(X) \sqsubseteq a s g^{\prime}(X)$ for all $X$ in $S V a r$. The meet and join are also defined pointwise. An abstract assignment asg represents a set of $\operatorname{sis}(\mathrm{T})$ structures as defined by the concretization conc $:$ Asg $g_{A} \rightarrow \mathcal{P}($ Struct $)$.

$$
\operatorname{conc}(\operatorname{asg}) \hat{=}\{(\tau, \sigma) \mid \text { for all } X \in S \operatorname{Var} .\{\tau(i) \mid i \in \sigma(X)\} \subseteq \gamma(\operatorname{asg}(X))\}
$$

An abstract assignment asg is a definite conflict for $\Phi$ if no model of $\Phi$ is in $\operatorname{conc}(a s g)$ and is a potential conflict if $\operatorname{conc}(\mathrm{asg})$ contains a countermodel of $\Phi$.

Trail. We introduce a trail, which contains meet-irreducibles as in $[4,10]$ and in which a second-order variable can appear multiple times. A trail over $A$ is the empty sequence $\epsilon$ or the concatenation $\operatorname{tr} \cdot(X: m)$, where $X$ is a second-order variable and $m$ is a complementable meet-irreducible. A trail $t r$ defines the assignment $[t r]$ where $[\epsilon] \hat{=} \lambda Y$. $\top$ and $[t r \cdot(X: m)]$ maps $X$ to $[t r](X) \sqcap m$ and all other $Y$ to $[t r](Y)$. A trail $t r$ is in potential/definite conflict with $\Phi$ if $[t r]$ is. We write $\operatorname{tr}(X)$ for $[\operatorname{tr}](X)$. An explanation exp for a trail of length $n$ is a function from $[0, n-1]$ to constraints in $\operatorname{Inf}_{G}$ or learnt clauses.

Search(). Algorithm 2 extends a trail $t r$ by propagating constraints from the CFG, making decisions, or applying a generalized unit rule. It returns divergent if $\operatorname{tr}\left(X_{\mathrm{ex}}\right)$ is $\perp$, meaning that ex is unreachable. It returns unknown if $\operatorname{tr}\left(X_{\mathrm{ex}}\right)$ is not $\perp$ and no decisions can be made. This trail is a potential conflict because every structure in $\operatorname{conc}([t r])$ with a non-empty assignment to $X_{\mathrm{ex}}$ violates the constraint $X_{\mathrm{ex}}(i) \Longrightarrow \operatorname{Last}(i)$, hence is a countermodel of $\operatorname{Inf} f_{G}$.

Example 3. The table alongside Algorithm 2 illustrates the construction of $t r$ and exp during interval analysis of the program in Fig. 2. The exp column shows the locations of the propagated constraints. The rows $1,2,3 a, 4 a, 5 a, 6 a$ represent a run of Search (). The trail is initially empty and the result of standard interval analysis is the trail $X_{\mathrm{ex}}:[-\infty, 0], X_{\mathrm{ex}}:[0, \infty]$ in step 2 , representing the assignment $\left\{X_{\text {in }} \mapsto \top, X_{\mathrm{a}} \mapsto \top, X_{\text {ex }} \mapsto[0,0]\right\}$. An arbitrary decision $X_{\text {in }}:[9, \infty]$ in step $3 a$ is not sound (see Example 3 ) and the smallest sound decision containing it is $[0, \infty]$. Propagation yields $X_{\mathrm{a}}:[1, \infty]$ in step $4 a$. The decision $X_{\text {in }}:[-\infty, 0]$ in step $5 a$ 

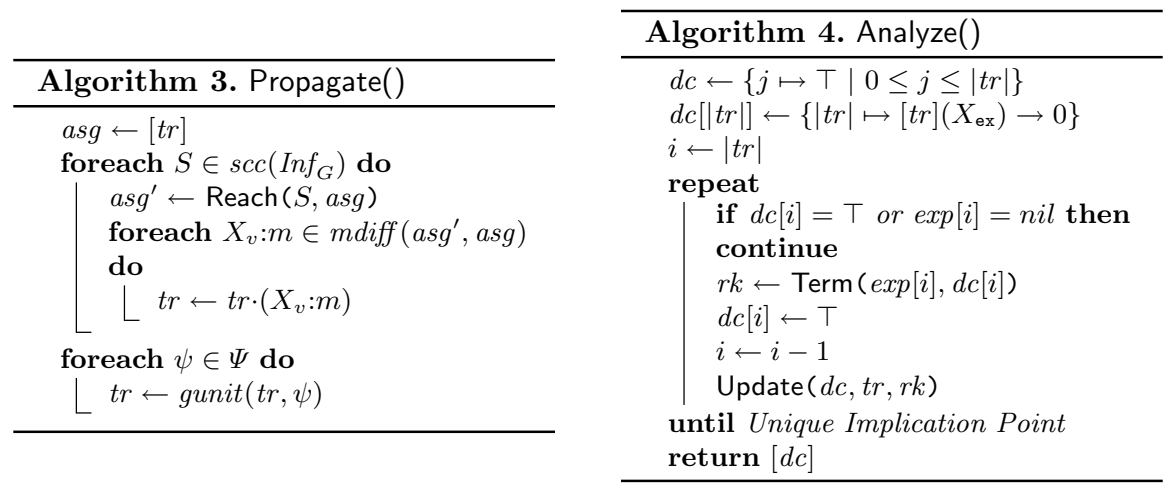

is sound, and when propagated, yields a conflict in step $6 a$, so search returns unknown. An alternative run is $1,2,3 b, 4 b$. A decision $X_{\text {in }}:[-\infty,-7]$ is sound, and propagation yields $X_{\mathrm{a}}:[-\infty,-7]$ and $X_{\mathrm{ex}}: \perp$, so search returns divergent. $\triangleleft$

Propagate(). Algorithm 3 calls an abstract interpreter and stores the results in the trail in a form amenable to conflict analysis and learning. The notion of meet-difference makes explicit the incremental change between two calls to the abstract interpreter. Formally, the meet-difference of $a, b \in A m \operatorname{diff}(a, b)=$ $m d c(a) \backslash m d c(b)$. The meet-difference of two abstract assignments is the pointwise lift $\operatorname{mdiff}\left(a s g, a s g^{\prime}\right)=\left\{X_{\mathrm{v}}: m \mid m \in \operatorname{mdiff}\left(\operatorname{asg}\left(X_{\mathrm{v}}\right), a s g^{\prime}\left(X_{\mathrm{v}}\right)\right), X_{\mathrm{v}} \in S \operatorname{Var}\right\}$.

In a transition constraint $\psi \hat{=} \forall i . \forall j . X_{\mathrm{v}}(j) \wedge S u c(i, j) \Rightarrow \ldots$, we write $\operatorname{sink}(\psi)$ for $X_{\mathrm{v}}$. A strongly connected component ( $\mathrm{SCC}$ ) of $I n f_{G}$ is a set of transition constraints $T$ such that the set of locations $\left\{v \mid \psi \in T, X_{\mathrm{v}}=\operatorname{sink}(\psi)\right\}$ is an SCC of $G$. The set of sCCs of $\operatorname{Inf}_{G}$ is $\operatorname{scc}\left(\operatorname{Inf} f_{G}\right)$. Propagate() calls a standard abstract interpreter on each SCC and uses a meet-difference calculation to extend the trail with new information. Propagate() also applies a generalized unit rule gunit, explained in $\S$ conflicts. Propagation is sound in the sense that it does not eliminate models of the constraints involved.

Lemma 1. If $(\tau, \sigma)$ satisfies $\operatorname{Inf}_{G}$ and $\Psi$ and is in conc $([\operatorname{tr}])$, it is also in $\operatorname{conc}([\operatorname{tr}])$ after invoking Propagate().

Decisions. The abstract assignment computed by (the abstract interpreter used by) Propagate() can be refined using decisions. Boolean decisions make variables true or false and first-order decisions use values $[7,24]$ but our decisions, like those in [11], use abstract domain elements.

A decision is an element $X: m$ that can be on a trail. A decision is sound if $\operatorname{conc}(X: m) \cup \operatorname{conc}(X: \bar{m})=$ Struct. That is, considering the structures in $m$ and $\bar{m}$ amounts to considering all possible structures.

Example 4. Recall the unsound decision $X_{\text {in }}:[9, \infty]$ from Example 3. The structure $(\tau, \sigma)$ with $\tau=9,9,8,8, \ldots$ and $\sigma$ partitioning $X_{\text {in }}$ and $X_{\mathrm{a}}$ into even and odd values is not in $\operatorname{conc}\left(X_{\text {in }}:[9, \infty]\right)$ as $x$ cannot be 8 at in. Similarly, it is not in $\operatorname{conc}\left(X_{\text {in }}:[-\infty, 8]\right)$ so $\operatorname{conc}\left(X_{\text {in }}:[9, \infty]\right) \cup \operatorname{conc}\left(X_{\text {in }}:[-\infty, 8]\right) \neq$ Struct. 
The unsoundness arises because pointwise lifting does not preserve concrete complements. Though $\bar{m}$ is the concrete complement of $m$ in $A,\left[X_{v}: \bar{m}\right]$ need not be the concrete complement of $\left[X_{v}: m\right]$ in $A s g_{A}$. Unsound decisions can be extended by propagation to a post-fixed point to cover all structures. All decisions on variables $X_{v}$ in singleton sCCs with no self-loops are sound.

A decision rule $\operatorname{dec}\left(\operatorname{Inf} f_{G}, \Psi, t r\right)$ returns an abstract domain element $d$ such that $\left[\operatorname{tr} \cdot\left(X_{v}: d\right)\right] \sqsubseteq[t r]$. The decision rule makes progress if this order is strict. Unlike in SAT the decision rule can cause divergence of CDCT because an infinite series of decisions like $[0, \infty],[1, \infty], \ldots$ may not change the result of propagation.

\section{Conflict Analysis}

Unlike SAT and SMT solvers, which generate definite conflicts, Search () generates potential conflicts. We apply backwards abstract interpretation with ranking functions to extract definite conflicts, and use widening to generalize them.

Ranking Function Domains. Due to space limitations, we only briefly recall the concrete domain of ranking functions, which provides the intuition for conflict analysis, and discuss the abstract domain informally. See [8,31] for details.

We write $f: A \nrightarrow B$ for a partial function whose domain is $\operatorname{dom}(f)$. A ranking function $f$ : State $\nrightarrow \mathbb{O}$ for a relation $R$ is a map from states to ordinals satisfying that for all $s$ in $\operatorname{dom}(f)$ and $(s, t)$ in $R, t$ is in $\operatorname{dom}(f)$ and $f(t)<f(s)$. A concrete domain for termination analysis $(\operatorname{Rank}, \preccurlyeq, B)$ is a lattice of ranking functions with backwards transformers $B=\left\{b k w_{c} \mid c \in C m d\right\}$ defined below. Informally $f \preccurlyeq g$ if $f$ is defined on a state when $g$ is and yields a lower rank: $f \preccurlyeq g \hat{=} \operatorname{dom}(f) \supseteq \operatorname{dom}(g)$ and for all $x$ in $\operatorname{dom}(g), f(x)<g(x)$. The transformer $b k w_{c}$ maps a ranking function $f$ to one defined on states with all their successors in $\operatorname{dom}(f)$. Recall that $\operatorname{Rel}_{c}$ is the transition relation for a command $c$.

$$
b k w_{c}(f) \hat{=} \lambda s \cdot \begin{cases}0 & \text { if } \operatorname{Rel}_{c}(s)=\emptyset \\ \sup \left\{f(r) \mid r \in \operatorname{Rel}_{c}(s)\right\}+1 & \text { if } \operatorname{Rel}_{c}(s) \subseteq \operatorname{dom}(f) \\ \text { undefined } & \text { otherwise }\end{cases}
$$

A subset $P \subseteq A$ of a domain $A$ is an abstract partition if $\{\gamma(a) \mid a \in P\}$ partitions State. Let Fun $\subseteq$ Rank be a lattice of functions, for example, affine functions.

A piecewise defined ranking function (PDRF) over Fun and $A$ is a set $\rho \hat{=}$ $\left\{a_{1} \mapsto f_{1}, \ldots, a_{k} \mapsto f_{k}\right\}$ such that $\left\{a_{1}, \ldots, a_{k}\right\}$ is an abstract partition, and each $f_{i}$ is in Fun. The abstract domain of PDRFs (aRank, $\left.\preccurlyeq, A b d\right)$ is a lattice aRank with abduction transformers Abd. The concretization $\gamma^{r}:$ aRank $\rightarrow$ Rank of a $\rho$ as above maps states to ranking functions: $\gamma^{r}(\rho) \hat{=}\left\{s \mapsto f_{i} \mid s \in \gamma\left(a_{i}\right)\right\}$. The order and lattice operations are defined in terms of partition refinement and unification [31]. To compare $\rho_{1}$ and $\rho_{2}$, we consider the coarsest abstract partition that refines the abstract partitions of both and compare the ranking functions in each block pointwise. 
Conflict analysis starts with a precondition for termination and finds a weaker precondition for termination, hence performs abduction. The abduction transformers satisfy the soundness condition: $\gamma^{r}\left(a b d_{c}(\rho)\right) \preccurlyeq b k w_{c}\left(\gamma^{r}(\rho)\right)$, which states that the termination bounds obtained with PDRFs are weaker than those that could be obtained in the concrete domain. A sound abduction transformer is underapproximating. A ranking assignment $r k:$ SVar $\rightarrow$ aRank associates a PDRF with each second-order variable. Ranking assignments form a lattice with point-wise meet and join and have a special order $\leqslant$ for fixed point checks [31]. To exchange information between Analyze() and Search () we extract a meetirreducible representation of the domains of PDRFs. The meet-projection of a $\operatorname{PDRF} \rho \hat{=}\left\{a_{i} \mapsto f_{i}\right\}$ is the set of sets of meet-irreducibles $\operatorname{mpr}(\rho) \hat{=}\left\{m d c\left(a_{i}\right)\right\}$ and provides a DNF-like representation of the abstract partition in $\rho$.

Analyze(). Algorithm 4 uses an array $d c$ to construct and generalize a definite conflict. Each $d c[i]$ represents termination conditions for states in the trail. Executions from states at ex terminate immediately so the last element of $d c$ is $\left\{X_{\mathrm{ex}} \mapsto\left\{[\operatorname{tr}]\left(X_{\mathrm{ex}}\right) \mapsto 0\right\}\right\}$ and all other elements are $\top$. The conflict analysis loop walks backwards through the trail and extends $d c[i]$. Forward propagation through the SCC $\exp [i]$ added $\operatorname{tr}[i]$ to the trail, so $d c[i]$ is propagated backwards through $\exp [i]$ to generalize the conflict to a ranking assignment $r k$. New PDRFs are added to $d c$ by the procedure Update(). Specifically, for each $X_{v}$ modified by Term (), and $m \in \operatorname{mpr}\left(r k\left(X_{v}\right)\right)$, Update() finds trail indices with $\operatorname{tr}[j] \sqsubseteq X_{v}: m$ and sets $d c[j]$ to the appropriate PDRF. Analyze() continues until a unique implication point is reached, which is typically a dominator in the CFG at which a decision was made. Analyze() returns $[d c]$, a representation of the PDRFs in $d c$.

Learn() and the Generalized Unit Rule. Information computed by Search() is communicated to Analyze() using the trail, while information from Analyze() is represented within Search () by a blocking constraint and is incorporated in search using generalized unit rule. We describe these very briefly.

A set $C=\left\{X_{1}: m_{1}, \ldots, X_{k}: m_{k}\right\}$ of elements can be complemented elementwise to obtain $\bar{C}=\left\{X_{1}: \bar{m}_{1}, \ldots, X_{k}: \bar{m}_{k}\right\}$. If $C$ is viewed as a conjunction of literals representing a conflict, $\bar{C}$ is a clause the procedure can learn. Learn() applies meet-projection to a PDRF and complements this projection to obtain a blocking constraint. In practice, we simplify the partitions of the PDRF to avoid an explosion of blocking constraints, analogous to subsumption in SAT.

The generalized unit rule [10] extends a trail using a blocking constraint. Assume that $\Psi$ has the form $\left\{X_{0}: m_{0}, \ldots, X_{k}: m_{k}\right\}$. The trail gunit $(t r, \Psi)$ is $t r$. $\left(X_{k}: m_{k}\right)$ if $[\operatorname{tr}]\left(X_{i}\right) \sqcap m_{i}=\perp$ for $0 \leq i<k$ and is $\operatorname{tr}$ otherwise. The generalized unit rule refines a trail in the sense that $[$ gunit $(t r, \Psi)] \sqsubseteq[t r]$. If $t r$ is inconsistent with $\Psi,[t r]$ will represent $\perp$. Having presented all components of the procedure, we now investigate how it works in practice.

\section{Implementation}

We have incorporated CDCT in our prototype static analyzer FUNCTION (http:// www.di.ens.fr/ urban/FuncTion.html), which is based on piecewise-defined 
(a)

\begin{tabular}{|c|c|c|c|}
\hline & Tot & Time & Timeouts \\
\hline \hline FuncTion+CDCT & 200 & $1.5 \mathrm{~s}$ & 15 \\
AProVE [29] & 256 & $15.9 \mathrm{~s}$ & 24 \\
FuncTion [32] & 175 & $0.7 \mathrm{~s}$ & 5 \\
HiPTnT+ [22] & 246 & $1.2 \mathrm{~s}$ & 4 \\
Ultimate [18] & 226 & $15.3 \mathrm{~s}$ & 35 \\
\hline
\end{tabular}

(b)

\begin{tabular}{|c|c|c|c|c|}
\hline & \multicolumn{4}{|c|}{ FuncTiON+CDCT } \\
& $\mathbf{0}$ & $\boldsymbol{\Delta}$ & $\times$ & \\
\hline AProVE [29] & 15 & 71 & 185 & 17 \\
FunCTION [32] & 25 & 0 & 175 & 88 \\
HIPTNT+ [22] & 22 & 68 & 178 & 20 \\
Ultimate [18] & 41 & 67 & 159 & 21 \\
\hline
\end{tabular}

Fig. 3. Overview of the experimental evaluation.

ranking functions [31]. A version without CDCT [32] participated in the 4th International Competition on Software Verification (SV-COMP 2015).

FUNCTION+CDCT accepts (non-deterministic) programs in a C-like syntax. It is implemented in OCaml and uses the APRON library [20]. The pieces of a PDRF can be represented with intervals, octagons or convex polyhedra, and ranking functions within the pieces are represented by affine functions. The precision of the analysis can also be controlled by adjusting the widening delay.

Experimental Evaluation. We evaluated our tool against 288 terminating C programs from the termination category of $S V$-COMP 2015. In particular, we compared FUNCTION+CDCT with other tools from the termination category of SV-COMP 2015: AProVE [29], FunCTION without CDCT [32], HIPTNT+ [22], and Ultimate Automizer [18]. The experiments were performed on a system with a $1.30 \mathrm{GHz}$ 64-bit Dual-Core CPU (Intel i5-4250U) and $4 \mathrm{~GB}$ of RAM. For the other tools, since we did not have access to their competition version, we used the $S V$-COMP 2015 results obtained on more powerful systems with a 3.40 GHz 64-bit Quad-Core CPU (Intel i7-4770) and 33 GB of RAM.

Figure 3 summarizes our evaluation. The first column is the number of programs each tool could prove terminating. The second column reports the average running time in seconds, and the last column reports the number of time outs, which was set to 180 seconds. In Fig. 3b, the first column (ם) lists the number of programs that FUNCTION+CDCT proved terminating and the tool could not, the second column $(\Delta)$ reports the number of programs that the tool proved terminating and FUNCTION+CDCT could not, and the last two columns report the number of programs that the tool and FUNCTION+CDCT were both able $(\times)$ or unable $(\bigcirc)$ to prove terminating. The same symbols are used in Fig. 4.

Figure 3 a shows that CDCT causes a $9 \%$ improvement in FUNCTION+CDCT compared to FUNCTION without CDCT. The increase in runtime is not evenly distributed, and about $2 \%$ of the test cases require more than 20 seconds to be analyzed by FunCTION+CDCT (cf. Fig. 4a). In these cases the decision heuristics do not quickly isolate sets of states on which the abstract interpreter makes progress. Figure 4 a shows that, as expected, FUNCTION without CDCT terminates with an unknown result earlier. Figures $4 \mathrm{~b}$ and $4 \mathrm{~d}$ show that though AProve and Ultimate Automizer were run on more powerful machines, FUNCTION+CDCT is generally faster but proves termination of respectively $19 \%$ and $9 \%$ fewer programs (cf. Fig. 3a). HIPTNT+ proves termination of $16 \%$ more 


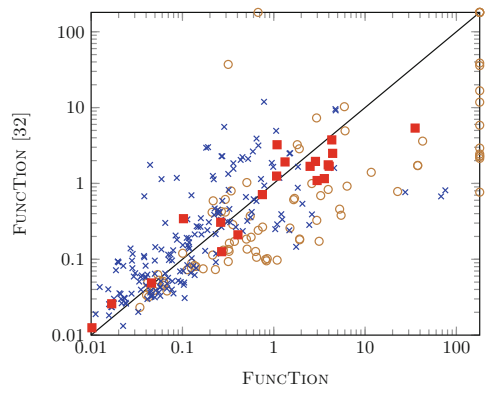

(a)

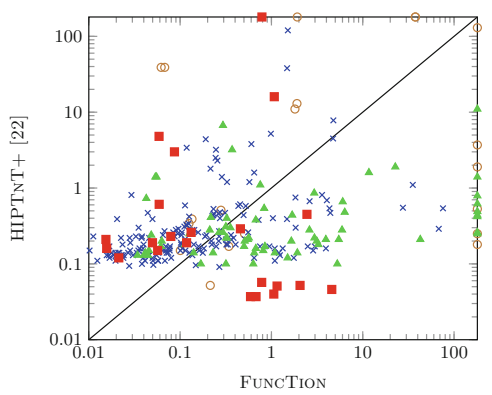

(c)

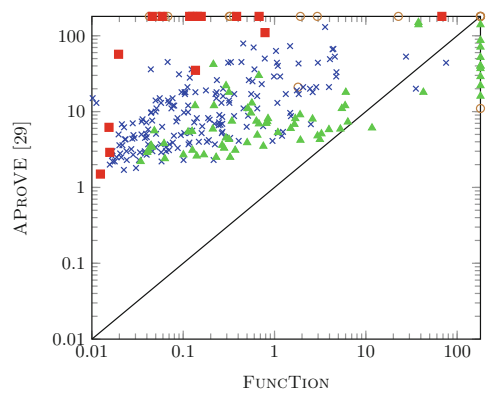

(b)

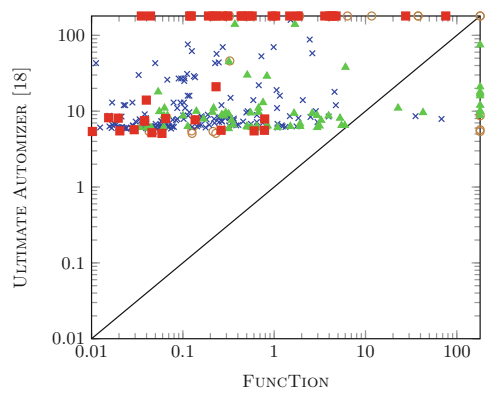

(d)

Fig. 4. Detailed comparison of FUnCTION against its previous version [32] (a), AProVe [29] (b), HiPTnT+ [22] (c), and Ultimate Automizer [18] (d).

programs than FunCTION+CDCT (cf. Fig. 4a), but FunCTION+CDCT proves termination of $52 \%$ of the program that HIPTNT + is not able to prove terminating ( $8 \%$ of the total test cases, cf. Fig. $3 \mathrm{~b})$. When comparing with FUNCTION without CDCT [32], we observed a 2x speedup in the $S V$-COMP 2015 machines, so the runtime comparison of FUNCTION+CDCT and HIPTNT+ is inconclusive. Finally, thanks to the support for piecewise-defined ranking functions, $1 \%$ of the programs could be proved terminating only by FUNCTION+CDCT $(2.7 \%$ by AProVE, $1 \%$ by HIPTnT+, and $1.7 \%$ by Ultimate Automizer). No tool could prove termination for $0.7 \%$ of the programs.

\section{Related Work and Conclusion}

Büchi's work relating automata and logic [5] is the basis for automata-based verification and synthesis. We depart from most work in this tradition in two ways. One is the use of sequences of first-order structures as in first-order temporal logics [19] and the other is to go from a graph-based representation to a formula, which is opposite of the translation used in automata-theoretic approaches. The use of s1s for pointer analysis [26], and termination [25] is restricted to decidable cases, as is [9]. Program analysis questions have been formulated with setconstraints [1] and second-order Horn clauses [13], but solutions to these formulae 
are typically invariants and ranking functions, not errors, and the methods used to solve them differ from CDCT.

A key intuition behind our work is to lift algorithmic ideas from SAT solvers to program analysis. The same intuition underlies SMPP [17], which lifts DPLL(T) to programs, ACDCL $[10,11]$, which lifts CDCL to lattices, the lifting of Stålmarck's method [30], and lazy annotation, which uses interpolants for learning [23]. The idea of guiding an abstract interpreter away from certain regions appears in DAGGER [14] and VINTA [2], from which CDCT differs in the use of a trail in search and a unit rule in learning. Our generalized unit rule is from ACDCL, but the use of $\operatorname{sis}(\mathrm{T})$, potential conflicts and the combination with PDRFs is all new. The widening used in CDCT preserves a termination guarantee and we believe that algorithms for generating small interpolants [3] can help design better widening operators.

Finally, termination analysis is a thriving area with more approaches than we can discuss. A fundamental problem is the efficient discovery of disjunctions of ranking functions [27]. We use backward analysis, as in [8,12], and our combination of conditional termination [6] with non-termination [15,21] is crucial. The approach of [22] is similar ours with a different refutation step and information exchange mechanism. At a high level, CDCT is the dual of [16], which underapproximates non-terminating executions and overapproximates terminating ones, while we overapproximate non-termination and underapproximate termination. We believe CDCT can be extended to transition-based approaches [28], but the challenge is to develop search and learning.

\section{References}

1. Aiken, A.: Introduction to set constraint-based program analysis. Sci. Comput. Program. 35, 79-111 (1999). Elsevier

2. Albarghouthi, A., Gurfinkel, A., Chechik, M.: Craig interpretation. In: Miné, A., Schmidt, D. (eds.) SAS 2012. LNCS, vol. 7460, pp. 300-316. Springer, Heidelberg (2012)

3. Albarghouthi, A., McMillan, K.L.: Beautiful interpolants. In: Sharygina, N., Veith, H. (eds.) CAV 2013. LNCS, vol. 8044, pp. 313-329. Springer, Heidelberg (2013)

4. Brain, M., D'silva, V., Griggio, A., Haller, L., Kroening, D.: Deciding floatingpoint logic with abstract conflict driven clause learning. Formal Methods Syst. Des. 45(2), 213-245 (2014). Springer

5. Büchi, J.R.: On a decision method in restricted second order arithmetic. In: Logic, Methodology and Philosophy of Science, pp. 1-11. Stanford University Press (1960)

6. Cook, B., Gulwani, S., Lev-Ami, T., Rybalchenko, A., Sagiv, M.: Proving conditional termination. In: Gupta, A., Malik, S. (eds.) CAV 2008. LNCS, vol. 5123, pp. 328-340. Springer, Heidelberg (2008)

7. Cotton, S.: Natural domain SMT: a preliminary assessment. In: Chatterjee, K., Henzinger, T.A. (eds.) FORMATS 2010. LNCS, vol. 6246, pp. 77-91. Springer, Heidelberg (2010)

8. Cousot, P., Cousot, R.: An abstract interpretation framework for termination. In: Field, J., Hicks, M. (eds.) POPL, pp. 245-258. ACM (2012) 
9. David, C., Kroening, D., Lewis, M.: Unrestricted termination and non-termination arguments for bit-vector programs. In: Vitek, J. (ed.) ESOP 2015. LNCS, vol. 9032, pp. 183-204. Springer, Heidelberg (2015)

10. D'Silva, V., Haller, L., Kroening, D.: Abstract conflict driven learning. In: Giacobazzi, R., Cousot, R. (eds.) POPL, pp. 143-154. ACM (2013)

11. D'Silva, V., Haller, L., Kroening, D., Tautschnig, M.: Numeric bounds analysis with conflict-driven learning. In: Flanagan, C., König, B. (eds.) TACAS 2012. LNCS, vol. 7214, pp. 48-63. Springer, Heidelberg (2012)

12. Ganty, P., Genaim, S.: Proving termination starting from the end. In: Sharygina, N., Veith, H. (eds.) CAV 2013. LNCS, vol. 8044, pp. 397-412. Springer, Heidelberg (2013)

13. Grebenshchikov, S., Lopes, N.P., Popeea, C., Rybalchenko, A.: Synthesizing software verifiers from proof rules. In: Vitek, J., Lin, H., Tip, F. (eds.) PLDI, pp. 405-416. ACM (2012)

14. Gulavani, B.S., Chakraborty, S., Nori, A.V., Rajamani, S.K.: Automatically refining abstract interpretations. In: Ramakrishnan, C.R., Rehof, J. (eds.) TACAS 2008. LNCS, vol. 4963, pp. 443-458. Springer, Heidelberg (2008)

15. Gupta, A., Henzinger, T.A., Majumdar, R., Rybalchenko, A., Xu, R.-G.: Proving non-termination. In: Necula, G.C., Wadler, P. (eds.) POPL, pp. 147-158. ACM (2008)

16. Harris, W.R., Lal, A., Nori, A.V., Rajamani, S.K.: Alternation for termination. In: Cousot, R., Martel, M. (eds.) SAS 2010. LNCS, vol. 6337, pp. 304-319. Springer, Heidelberg (2010)

17. Harris, W.R., Sankaranarayanan, S., Ivančić, F., Gupta, A.: Program analysis via satisfiability modulo path programs. In: Hermenegildo, M., Palsberg, J. (eds.) POPL, pp. 71-82. ACM (2010)

18. Heizmann, M., Dietsch, D., Leike, J., Musa, B., Podelski, A.: Ultimate Automizer with array interpolation (Competition Contribution). In: Baier, C., Tinelli, C. (eds.) TACAS 2015. LNCS, vol. 9035, pp. 455-457. Springer, Heidelberg (2015)

19. Hodkinson, I.M., Wolter, F., Zakharyaschev, M.: Decidable and undecidable fragments of first-order branching temporal logics. In: LICS, pp. 393-402. IEEE Computer Society (2002)

20. Jeannet, B., Miné, A.: Apron: a library of numerical abstract domains for static analysis. In: Bouajjani, A., Maler, O. (eds.) CAV 2009. LNCS, vol. 5643, pp. 661667. Springer, Heidelberg (2009)

21. Larraz, D., Nimkar, K., Oliveras, A., Rodríguez-Carbonell, E., Rubio, A.: Proving non-termination using max-SMT. In: Biere, A., Bloem, R. (eds.) CAV 2014. LNCS, vol. 8559, pp. 779-796. Springer, Heidelberg (2014)

22. Le, T.-C., Qin, S., Chin, W.-N.: Termination and non-termination specification inference. In: Grove, D., Blackburn, S. (eds.) PLDI. ACM (2015)

23. McMillan, K.L.: Lazy annotation for program testing and verification. In: Touili, T., Cook, B., Jackson, P. (eds.) CAV 2010. LNCS, vol. 6174, pp. 104-118. Springer, Heidelberg (2010)

24. McMillan, K.L., Kuehlmann, A., Sagiv, M.: Generalizing DPLL to richer logics. In: Bouajjani, A., Maler, O. (eds.) CAV 2009. LNCS, vol. 5643, pp. 462-476. Springer, Heidelberg (2009)

25. Mesnard, F., Payet, É.: A second-order formulation of non-termination. In: CoRR (2014)

26. Møller, A., Schwartzbach, M.I.: The pointer assertion logic engine. In: Burke, M., Soffa, M.L. (eds.) PLDI, pp. 221-231. ACM (2001) 
27. Podelski, A., Rybalchenko, A.: Transition invariants. In: LICS, pp. 32-41. IEEE Computer Society (2004)

28. Podelski, A., Rybalchenko, A.: Transition invariants and transition predicate abstraction for program termination. In: Abdulla, P.A., Leino, K.R.M. (eds.) TACAS 2011. LNCS, vol. 6605, pp. 3-10. Springer, Heidelberg (2011)

29. Ströder, T., Aschermann, C., Frohn, F., Hensel, J., Giesl, J.: AProVE: Termination and memory safety of $\mathrm{C}$ programs (Competition Contribution). In: Baier, C., Tinelli, C. (eds.) TACAS 2015. LNCS, vol. 9035, pp. 417-419. Springer, Heidelberg (2015)

30. Thakur, A., Reps, T.: A generalization of Stålmarck's method. In: Miné, A., Schmidt, D. (eds.) SAS 2012. LNCS, vol. 7460, pp. 334-351. Springer, Heidelberg (2012)

31. Urban, C.: The abstract domain of segmented ranking functions. In: Logozzo, F., Fähndrich, M. (eds.) Static Analysis. LNCS, vol. 7935, pp. 43-62. Springer, Heidelberg (2013)

32. Urban, C.: FuncTion: an abstract domain functor for termination (Competition Contribution). In: Baier, C., Tinelli, C. (eds.) TACAS 2015. LNCS, vol. 9035, pp. 464-466. Springer, Heidelberg (2015) 